PROFESIONALES Y HERRAMIENTAS PARA EL DESARROLLO LOCAL Y SUS SINERGIAS TERRITORIALES. EVALUACIÓN Y PROPUESTAS DE FUTURO IX Coloquio Nacional de Desarrollo Local del GTDL-AGE 

ANTONIO MARTÍNEZ PUCHE, XAVIER AMAT MONTESINOS, ISABEL SANCHO CARBONELL y DANIEL SANCHIZ CASTAÑO (EDS.)

\section{PROFESIONALES Y HERRAMIENTAS PARA EL DESARROLLO LOCAL Y SUS SINERGIAS TERRITORIALES. EVALUACIÓN Y PROPUESTAS DE FUTURO}

IX Coloquio Nacional de Desarrollo Local del GTDL-AGE

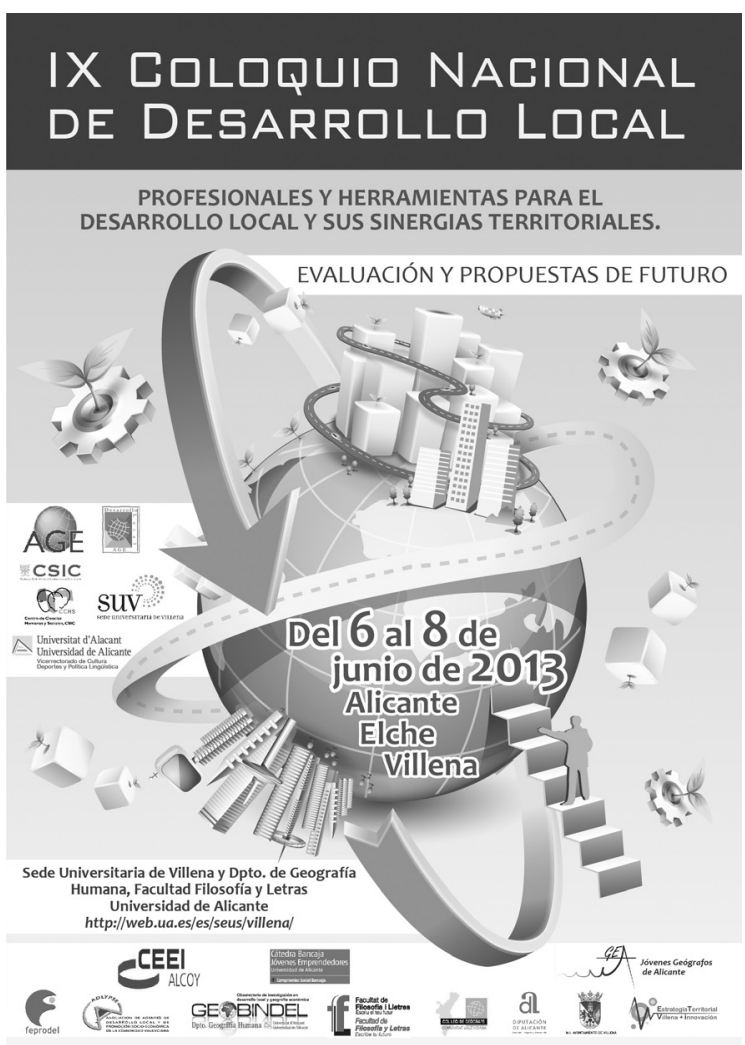


Este libro ha sido examinado y valorado por evaluadores ajenos a la Universidad de Alicante, con el fin de garantizar la calidad científica del mismo.

Publicacions de la Universitat d'Alacant

03690 Sant Vicent del Raspeig

Publicaciones@ua.es

http://publicaciones.ua.es

Telèfon: 965903480

(C) Antonio Martínez Puche, Xavier Amat Montesinos,

Isabel Sancho Carbonell y Daniel Sanchiz Castaño (eds.), 2016

(C) d'aquesta edició: Universitat d'Alacant

ISBN: 978-84-16724-00-0

Dipòsit legal: A 92-2016

Disseny de coberta: candela ink

Composició: Página Maestra (Miguel Ángel Sánchez Hernández)

Impressió i enquadernació: Guada Impresores

\section{unte \\ Unión de Editoriales
Universitarias Españolas \\ WWW.une.es
WWA}

Esta editorial es miembro de la UNE, cosa que garantiza la difusión y comercialización nacional y internacional de sus publicaciones.

Reservados todos los derechos. Cualquier forma de reproducción, distribución, comunicación pública o transformación de esta obra sólo puede ser realizada con la autorización de sus titulares, salvo excepción prevista por la ley. Diríjase a CEDRO (Centro Español de Derechos Repográficos, www.cedro.org) si necesita fotocopias o escanear algún fragmento de esta obra. 


\title{
¿ESTÁN EL PELIGRO LOS AEDLs?
}

\author{
Oriol Estela Barnet \\ Diputación de Barcelona \\ estelabo@diba.cat
}

\begin{abstract}
RESUMEN
El panorama de cambios en la financiación de las políticas de desarrollo económico local y de reorganización de competencias vigente en España han introducido nuevas incertidumbres sobre el futuro de los Agentes de Empleo y Desarrollo Local (AEDL). Una adecuada reubicación de determinadas políticas de desarrollo económico local en una esfera supramunicipal, junto con la asunción del papel de dinamizador comunitario del AEDL a escala municipal son las claves para garantizar la continuidad de unos profesionales que contribuyen notablemente, en general, a la mejora del bienestar en su territorio.
\end{abstract}

Palabras clave: Desarrollo económico local, AEDL, competencias, dinamización comunitaria, bienestar.

\section{ARE THEY THE DANGER OF AEDLs?}

\section{AbSTRACT}

The changes in funding policies of local economic development and reorganisation of municipal competences existing in Spain have introduced new uncertainties about the future of Employment and Local Development agents (ELDA). A proper relocation of certain policies of local economic development in a supra-municipal sphere, along with the assumption of the role of community revitalization of the ELDA at the municipal level are the keys to ensuring the continuity of those professionals who contribute substantially, in general, to the improvement of welfare in its territory.

Key words: Local economic development, ELDA, competences, community revitalization, welfare 


\section{LOS AEDLS HOY ¿UNA CUESTIÓN DE RECURSOS Y COMPETENCIAS?}

La figura del Agente de Empleo y Desarrollo Local, nacida a mediados de los años 80 de la mano del extinto Instituto Nacional de Empleo, se encuentra en la actualidad, tras años de despliegue por toda la geografía española, en un momento crítico. No se trata ya de los problemas que han ido asociados a la precariedad del empleo resultado de su vinculación con programas de subvención anuales; o a la endémica indefinición del perfil profesional y las tareas a realizar en la teoría pero, sobre todo, en la práctica (el AEDL como único técnico cualificado en muchos municipios que le convertía en un útil comodín); ni siquiera a los cambios en el "mercado" del desarrollo económico local, que han supuesto la proliferación de organizaciones públicas, privadas y del tercer sector dedicadas al rosario de actividades que supuestamente debería realizar un AEDL.

Lo que condiciona el momento actual del debate sobre el papel de los AEDLs dentro del marco de las políticas locales, así como también sobre las propias políticas de empleo y desarrollo local, es una doble amenaza que puede conducir a su práctica desaparición, o al menos a una fuerte reconversión, dentro del panorama de las actuaciones de los gobiernos locales.

La primera amenaza proviene de la reducción, considerablemente drástica en algunos lugares, de los fondos disponibles por parte de las Comunidades Autónomas para financiar el programa de AEDLs en los últimos años. Casos como el de las Illes Balears, donde se ha pasado de un máximo histórico de alrededor de 110 AEDLs en el año 2010 a 0 en el año 2013 son un claro ejemplo del impacto de dicha reducción.

La relación causal entre reducción de fondos de los programas de las administraciones autonómicas y el número de AEDLs en el territorio es, por tanto, evidente. Sin embargo, el problema no debe tanto achacarse a la reducción en sí como a la incapacidad de los propios entes locales para integrar la figura del AEDL dentro de su estructura orgánica (que no funcional, puesto que tareas relacionadas con su ámbito de actuación nunca les han faltado). Los problemas endémicos de financiación de los gobiernos locales y la falta de competencias específicas explican una parte importante de la situación. Pero también la ya mencionada indefinición del perfil y funciones del AEDL, el hecho de asumirlo como algo externo y, por tanto, no proporcionarle el carácter estratégico que debería tener y la falta de articulación de redes supralocales que le dieran más fuerza a esta figura, no son factores menores.

Dicho de otro modo, la falta de recursos externos es un problema, sí, pero debería serlo menos de existir voluntad política, y un modelo y unas estrategias de desarrollo económico por las que el gobierno local apostara firmemente. Siempre y cuando, evidentemente, disponga de la capacidad para hacerlo. Algo que hoy en día se encuentra en entredicho, y aquí nos encontramos con la se- 
gunda amenaza, si atendemos al espíritu de la reforma de la Ley de Bases del Régimen Local que se encuentra en la antesala de su aprobación.

Ante estas amenazas, cabe preguntarse de nuevo por el sentido de la figura del AEDL, su perfil y sus actividades, de manera que nos sea posible identificar el espacio en el que dicha figura debe operar y, muy especialmente, aventurar si la falta de recursos y de competencias, algo que de por sí liquidaría la capacidad de acción de cualquier administración en cualquier terreno, van a significar el fin de de sus servicios para con la colectividad.

\section{2. ¿QUÉ DICEN LOS PROFESIONALES?}

A lo largo de los años, los AEDLs se han identificado notablemente con dos visiones altamente ejecutivas de las políticas de desarrollo local, que explicaremos a continuación de forma muy breve y necesariamente incompleta, pero ilustrativa.

Por una parte, se trataba de los encargados de llevar a buen puerto la ejecución de los programas que, más allá del que servía para financiar su contratación, conseguía la administración local para la que trabajaban. La imagen del AEDL tratando de alcanzar los indicadores que a priori se establecían en estos programas y su dedicación permanente a la elaboración de informes de seguimiento y similares puede ser una caricatura, en algunos casos lamentablemente real, de esta vertiente de su trabajo.

Por otra parte, llegó un momento que entre sus misiones se destacaba la "creación de empresas y de empleo", situando sobre sus hombros la pesada carga de corregir lo que ni la economía ni la sociedad locales tenían a bien generar.

Ambas vertientes, dominantes en la asignación de un rol específico a los AEDLs en las políticas locales, vienen determinadas, por tanto, por la existencia de unos programas (y sus correspondientes fondos) procedentes de otras administraciones y por el objetivo -y casi diríamos la responsabilidad- de encontrar un empleo a las personas que no lo tuvieren y de convertir en persona emprendedora-empresaria a todo aquél/la que mostrara un mínimo de aptitudes o cuando menos predisposición para ello. Algo que, para lo que nos atañe en este artículo, entra claramente en un ámbito competencial reservado en principio, según la letra de la ley, a la administración autonómica.

La celebración en Alicante del IX Coloquio Nacional de Desarrollo Local ha sido una buena oportunidad para tomar el pulso de los profesionales del sector acerca de la visión actual de la figura del Agente de Empleo y Desarrollo Local. Lógicamente, no se trataba de realizar un ejercicio con rigor científico si no simplemente tratar de obtener alguna información adicional de primera mano que permitiera detectar el espacio que pueden ocupar los AEDLs en estos tiempos de cambio. 
Con tal motivo, se distribuyeron unas tarjetas entre todos los asistentes a la primera jornada del Coloquio con la siguiente indicación: "Por favor, completa sólo una de las dos frases: a) Un agente de desarrollo local es... b) La tarea más importante que debe llevar a cabo un AEDL es... ¡MUCHAS GRACIAS!.

Se recibieron 39 tarjetas cumplimentadas. Las respuestas se han trascrito en el anexo 1. En 14 de las tarjetas se había dado respuesta a la primera de las preguntas, en 12 a la segunda y, contraviniendo las indicaciones, 13 personas optaron por contestar ambas.

El sentido de proponer la limitación a una respuesta residía en comprobar en qué medida los profesionales se sentían más cómodos describiendo la figura del AEDL bien por su perfil o bien por su función. El resultado, aparentemente, muestra indiferencia al respecto, ya que el número de respuestas en cada caso es similar. No obstante, la lectura de las respuestas muestra cómo, incluso entre quienes únicamente respondieron a la primera de las preguntas, el enfoque se correspondía de manera muy clara más con una descripción de tareas que con una descripción de características. Ello podría ser un indicador de la ya comentada indefinición (o, en otros términos, de la amplitud) del perfil del AEDL.

En la figura 1 se muestra una "nube de términos" que muestra las palabras más utilizadas para tratar de describir "qué es" un AEDL. El tamaño de letra es proporcional al número de respuestas obtenidas conteniendo cada uno de los términos. Se observa, así, que la idea más repetida es la del AEDL como "dinamizador", seguida de otras de significado parecido como "facilitador", "promotor" o "catalizador", todas ellas, como ya se ha indicado, más asociables a la tarea a realizar que al perfil del AEDL en sí mismo. En cualquier caso, lo más relevante de este parte del "experimento" es que se constata una identificación del AEDL con su tarea de articulación de personas y de recursos en el territorio. En definitiva, de construcción e impulso de redes para el desarrollo local.

Realizando el mismo ejercicio con las respuestas a la segunda pregunta (ver Figura 2) podemos llegar a una conclusión similar. Si bien la idea de "dinamizar" aparece expresada de esta forma con menos fuerza que en el caso anterior, algunos de los términos más utilizados -que muestran, por cierto una mayor diversidad y dispersión- son bastante consistentes con dicha idea: "promover", "organizar", "contribuir". A ello se añade la atribución de las tareas de conocimiento y análisis de la realidad del territorio, imprescindible para ejercer el papel dinamizador atribuido: "conocer", "identificar", "analizar"... Y aparece aquí también la idea de "crear": el AEDL debe utilizar su conocimiento y capacidad dinamizadora para obtener un valor para el territorio en el que opera. 


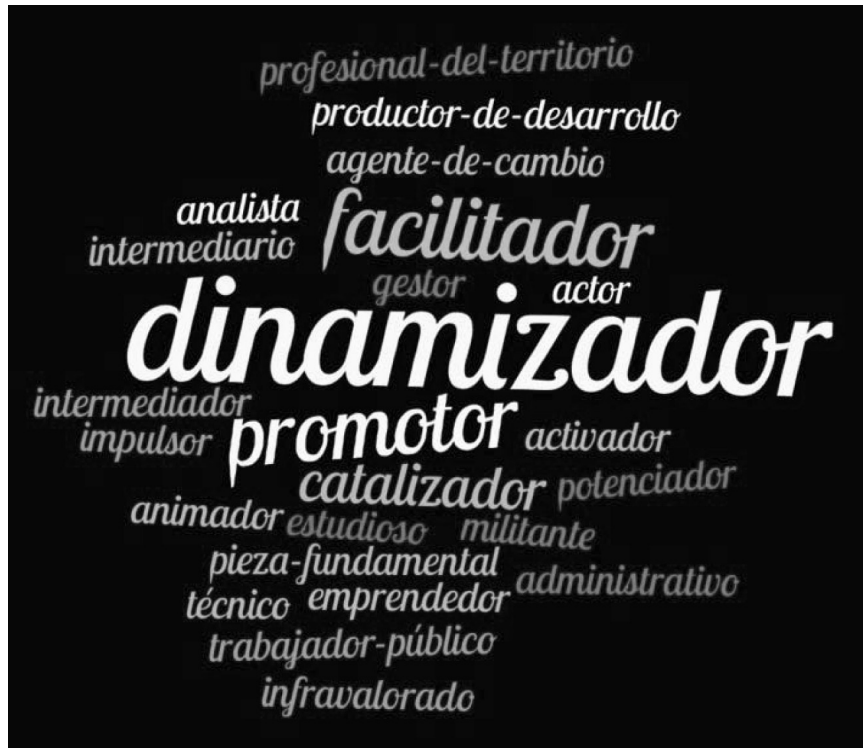

Figura 1: Un agente de desarrollo local es...

Fuente: elaboración propia.

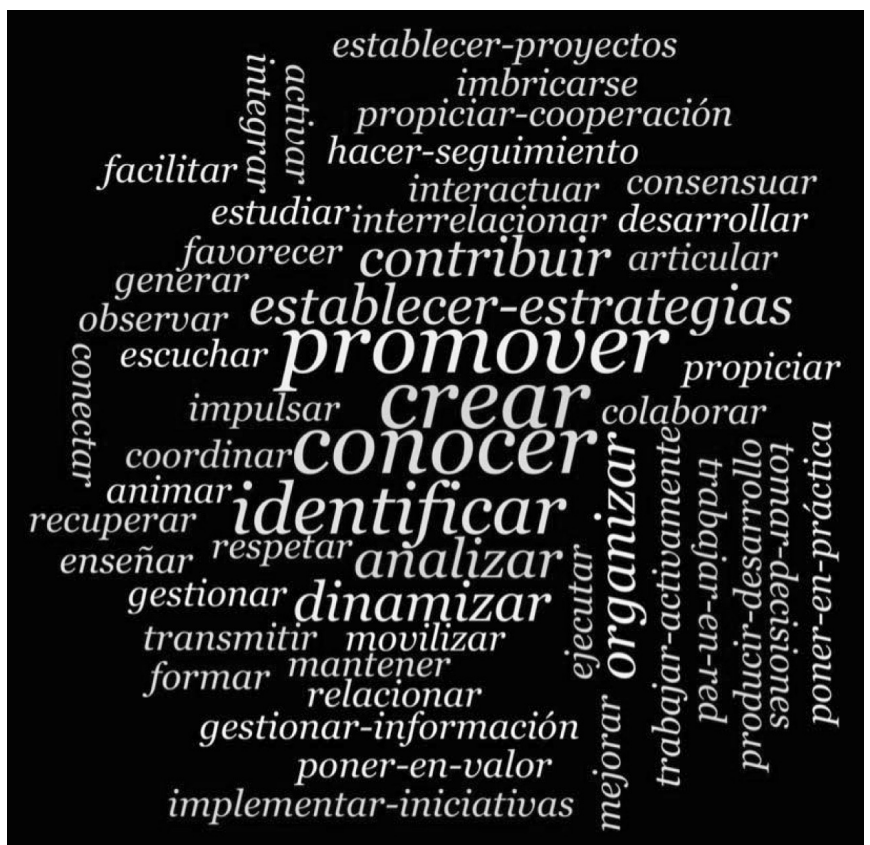

Figura 2: La tarea más importante que debe llevar a cabo un AEDL es...

Fuente: elaboración propia. 


\section{CONCLUSIÓN}

La evolución que ha experimentado el perfil del AEDL, tanto en el discurso como en la práctica (que no en su definición, invariable dentro de la falta de concreción), muestra el paso de un recurso técnico local orientado a la gestión y ejecución de programas predeterminados a una figura clave en la dinamización socioeconómica y la creación de redes.

Este cambio en, podríamos decir, el "imaginario" del AEDL es de suma importancia en el momento actual, en el que se produce la doble amenaza de los recortes en la financiación de los programas con los que se encuentra vinculada dicha figura y en el que se plantean unas limitaciones sustanciales en el alcance de las competencias municipales.

El impacto de la menor (o nula, según el caso) disponibilidad de fondos para financiar la contratación de AEDLs es y será muy importante, pero debería relativizarse si tenemos en cuenta la "anomalía" que representa que los entes locales hayan tenido a su disposición durante casi 30 años un recurso financiado externamente, siendo la asunción de dicho recurso de manera interna la excepción más que la regla. La voluntad y el convencimiento del estamento político acerca de la necesidad de disponer de recursos (económicos, técnicos y humanos) propios en materia de desarrollo económico local debería contrarrestar, en la medida en la que las castigadas arcas locales lo permitan, el abandono de este tipo de políticas por parte de las administraciones superiores.

Sin embargo, el enfoque de la reforma prevista de las bases del régimen local incide precisamente en la capacidad de los gobiernos locales para traducir voluntad y convencimiento político en actuaciones efectivas. El encorsetamiento competencial que se prevé conduce a una eliminación en la práctica de las políticas de desarrollo local desde los entes locales, que estarán sólo al alcance de municipios perfectamente saneados y con una actitud poco conservadora por parte de sus interventores/as. Serán, pues, gobiernos autonómicos, diputaciones y a lo sumo comarcas, allí donde estén institucionalizadas, los espacios de las políticas de desarrollo local. El desarrollo económico local, en definitiva, sin la participación de lo local-municipal.

A menos que seamos capaces de repensar y reexplicar el desarrollo económico local.

Efectivamente, el desarrollo económico local del modelo de los 80, basado en la captación de inversiones externas, puede encontrar un mejor acomodo en una escala superior, quizás la regional (en términos geográficos, no administrativos; es decir, la supramunicipal). Por su parte, el desarrollo económico local impulsado a partir de los 90, el del fomento de la emprendeduría, los distritos industriales-clusters-sistemas productivos locales o, más recientemente, los ecosistemas innovadores, también precisa de una masa crítica suficiente para llegar a conseguir resultados relevantes. Todas estas políticas, en las que 
los AEDLs participan de manera intensa (especialmente en las relacionadas con personas emprenedoras y articulación del tejido productivo local) pueden organizarse perfectamente, del mismo modo que los servicios locales de empleo, desde un nivel meso, sea metropolitano, comarcal o más genéricamente, supramunicipal. El nivel al que aparentemente las empuja de todas formas la reforma legislativa en ciernes. Ese sería, por tanto, el espacio reservado para los AEDLs en su sentido "clásico".

¿Pero qué sucede con el desarrollo local visto desde la perspectiva desde la que lo ven sus propios protagonistas? Esta perspectiva de la dinamización, del agente catalizador y promotor, del facilitador que conoce su entorno y contribuye a crear valor en el territorio nos muestra una dimensión del desarrollo económico local que poco tiene que ver con una competencia determinada. Sus vínculos son más directos con ámbitos como el del desarrollo comunitario o participativo que con lo que se podría entender como políticas activas de empleo o políticas de promoción económica al uso. Se trata de una perspectiva que, en realidad, obedece a una lógica diferente a la de las competencias, y mucho menos al de su reparto entre niveles distintos de la administración. Se trata de una perspectiva que se corresponde con la esencia misma del gobierno local y de la democracia local. Algo que ningún texto legislativo podrá eliminar de la lista de tareas inherentes al nivel de gobierno más próximo a la ciudadanía.

Así pues, en la medida en la que sepamos reorganizar las políticas de desarrollo económico local y su relato alrededor del fortalecimiento de la capacidad del territorio para generar su propio desarrollo desde un punto de vista más bien holístico, y en la medida en la que todo ello se desligue de la rigidez autoimpuesta de los programas mediante los que nos hemos venido financiando en los 30 años de existencia de este ámbito profesional, la figura del AEDL o como quiera llamársele en cada momento y en cada lugar, seguirá resultando imprescindible para mejorar la capacidad de los gobiernos locales para atender las necesidades y las aspiraciones de su población. 


\section{ANEXO}

Resultado de la consulta a las personas participantes en el IX Coloquio Nacional de Desarrollo Local.

Un agente de desarrollo local es...

- Un/a facilitador/a, en el amplio sentido del término. Aquél/lla que piensa en cómo encajar todos los elementos que pueden contribuir al desarrollo local.

- Un facilitador.

- Militante del territorio.

- El dinamizador del territorio. "En teoría", ya que en la práctica se podría reducir su labor meramente administrativa: la solicitud de subvenciones, justificación, etc... Abandonando su papel dinamizador.

- Un estudioso del territorio para producir desarrollo a partir de sus recursos.

- Profesionales que facilitan herramientas a empresarios, emprendedores, desempleados y ciudadanos en general para mejorar su situación económica y social.

- Aquel intermediario que ayuda a mejorar y a recuperar la evolución territorial.

- Una persona que trabaja por el equilibrio de un territorio en sus niveles económico, social y medioambiental. Analizando primero el medio, de modo que la planificación para su desarrollo sea apropiada y con un sentido duradero en el tiempo. Es el catalizador que aprovecha recursos y potencia capacidades territoriales y sociales. Es en muchos casos una persona que desarrolla una labor crucial que suele ser infravalorada.

- Quien trabaja para activar los recursos locales y convertir los genéricos en específicos, además de propiciar la cooperación público-privada para avanzar más en los procesos de desarrollo.

- El individuo o colectivo que se encarga de interactuar con la sociedad local y gestiona la información para desarrollar iniciativas de desarrollo local.

- Un animador territorial que logra (o intenta lograr) una concertación de actores relevantes para consensuar acciones locales para el desarrollo y el empleo.

- Un profesional del territorio encargado de identificar los recursos locales y crear estrategias innovadoras que contribuyan a la cohesión social, la dinamización económica y a la estructuración territorial.

- Una persona que se encarga de establecer y ejecutar proyectos para contribuir a una Comunidad.

- Una profesión que se encarga de llevar a cabo planes que beneficien o ayuden al desarrollo de una comunidad.

- Trabajador público al servicio del pueblo que promueve y organiza operaciones de promoción de desarrollo, integrar recursos culturales, económicos y naturales de la región. 
- Un actor que conoce el territorio, sus necesidades y potencialidades y realiza estrategias para llevar a cabo su desarrollo.

- Alguien que dinamiza políticas para el avance económico, social, cultural, turístico... de un territorio.

- El que impulsa el bienestar del ciudadano en el ámbito local.

- Pieza fundamental del complejo engranaje que es el desarrollo local.

- Dinamizador del territorio.

- Dinamizador, promotor y agente de cambio para articular los actores sociales en beneficio del territorio y su vocación.

- Un dinamizador, catalizador de las iniciativas locales.

- Un facilitar, promotor y dinamizador de las economías locales.

- Un gestor del desarrollo.

- Intermediador entre los diferentes agentes locales y dinamizador.

- Un técnico insertado en su entorno territorial y social, con mentalidad emprendedora que recoge o promueve iniciativas locales.

- Un dinamizador socio-económico.

La tarea más importante que debe llevar a cabo un AEDL es...

- Escuchar y animar a la acción.

- Movilizar todos los recursos disponibles en un territorio, sobre todo los humanos, de cara a avanzar en su [ilegible] de desarrollo local.

- Promoción socioeconómica.

- Estudio del entorno. Conexión de recursos. Relación con actores. Dinamización. "Enseñanza" a políticos.

- Dinamitzar recursos i territoris des de l'observació de la realitat o context i amb respecte als agents locals, cultura, idiosincrasia, medi ambient, recursos naturals...

- Promover procesos de participación ciudadana y concertación social entre agentes del territorio para impulsar proyectos de desarrollo local.

- Imbricarse en la sociedad local, conocerla desde dentro, respetarla y contribuir a su cambio desde la autonomía y la autorregulación.

- Trabajar activamente con diversos agentes socioeconómicos a fin de plantear y gestionar respuestas al contexto actual.

- Conocer las debilidades del ámbito local y [ilegible].

- Favorecer la creación de riqueza endógena a partir del establecimiento de estrategias pertinentes.

- Conocer el tejido productivo y los actores tecnológicos y formativos para hacer seguimiento de sus planes de desarrollo, potencialidades y vías de competitividad.

- La interrelación de elementos físicos, humanos y sociales que se dan en un espacio local; y la toma de decisiones como compendio de ese análisis. 
- El conocimiento profundo del territorio de actuación (conocer su entorno y los agentes socioeconómicos que lo componen).

- Colaborar con el desarrollo e implementación de iniciativas para el desarrollo local.

- Facilitar los procesos de los actores del territorio.

- Identificar, analizar y transmitir los recursos materiales e inmateriales para el desarrollo local. Coordinar acciones y relaciones de los distintos ámbitos.

- Crear tejido social.

- Identificar la vocación del territorio. Articular actores. Generar transformación social positiva de impacto territorial.

- Poner en valor la importancia de tener una buena estrategia de desarrollo local.

- Promover la innovación en el territorio.

- Mantener la red de actores locales y crear las sinergias necesarias entre ellos.

- Poner en práctica (y formar a los diferentes actores locales) metodologías e iniciativas innovadoras y sostenibles. Trabajar en red.

- Organizar a las entidades privadas y públicas para implementar las actividades de desarrollo local: crear redes y capital social.

- Propiciar el marco/contexto que permita la interlocución entre todos los actores públicos/privados envueltos en los procesos de desarrollo. 